\title{
Selection of compression test images using variance-based statistical method
}

\author{
Allaoui Chems El houda, Bassou Abdesselam, Benyahia Ismahane, Khelifi Mustapha \\ Department of Electrical Engineering, Tahri Mohammed University-Bechar, Algeria
}

\begin{tabular}{l} 
Article Info \\
\hline Article history: \\
Received Jan 10, 2019 \\
Revised Feb 12, 2019 \\
Accepted Mar 15, 2019 \\
\hline
\end{tabular}

\begin{abstract}
In this research paper, we used a variance-based statistical method to select 20 test images, according to these latter types (natural, satellite and medical) among a sample of 300 images (100 natural, 100 satellite and 100 medical). The images selection has been done using parameters of image quality, namely PSNR, MSSIM and VIF, which were applied on three compression algorithms (DWT+SPIHT, JPEG, and JPEG2000).
\end{abstract}

\section{Keywords:}

Discrete Wavelet Transform

Evaluation Parameters

Image compression

JPEG

JPEG2000

Selection Algorithm

SPHIT

Copyright (ㅇ 2019 Institute of Advanced Engineering and Science. All rights reserved.

\section{Corresponding Author:}

Allaoui Chems El houda,

Department of National Chung,

Tahri Mohammed University-Bechar, Algeria.

Email: chems66@hotmail.com

\section{INTRODUCTION}

JPEG (Joint Photographic Experts Group) standard has been invented in 1986 by ISO (International Standards Organization) group and CEI (Commission Electronic International) group. It was aimed at compressing fixed color images at grey-scale data in order to save them on numerical support. One of common problems related to JPEG are mosaic appearances when compression levels are higher. This pushed researchers to search for a new standard to compress images: JPEG2000, which uses updated knowledge in the field of wavelets technology. At the heart of JPEG standard, we have discrete wavelet transform (DWT), while JPEG2000 heart consists of a breaking down into wavelets to allow a hierarchical representation of images in order to structure data efficiently, especially linking spectral content to a spatial position of the picture signal [1].

Other image compression algorithms, based on DWT, have been proposed in letterature. For example, the binary encoding algorithm named Set Partitionning In Hierarchical Tree (SPIHT) [2] was associated to the DWT in order to generate the DWT+SPIHT compression algorithm; this compression method was inverstigated on fix and motion images, and produce a suitable image quality according to [3].

The diversity of these compression algoritms has pushed us to propose a set of test images that permits to validate the effiency of future algorithms of image compression. The proposed images set is obtained by proposing a selection algorimth based on variance-based statistical method applied on image quality evaluation parameters. 


\section{METHODS OF IMAGES COMPRESSION}

\subsection{JPEG Transform}

JPEG is a sophisticated compression method with or without losses whether at grey-scales or in color image. Such a standard does not handle monochromatic compression. It works for continuous tone images as well. One of the advantages of JPEG standard is that it uses many parameters which enable users to adjust lost data (i.e. compression level). Here are the main JPEG coding steps:

a) Preparation

b) TCD (Transform in Discrete Cosine) on $8 \times 8$ units

c) Quantification

d) Zigzag reading

e) DC component direct coding (average of $8 \times 8$ unit)

f) Coding by range of AC component (RLE: Run Length Encoding)

g) Entropic coding of Huffman [4] type (variable length coding: VLC).

For very high compression coding, we prefer compressing with wavelets or fractals to avoid edge effect which takes place sometimes under the shape of color rings near the remaining clear outlines.

\subsection{JPEG 2000 Transform}

JPEG 2000 is by its very nature multi-resolution standard, this allows us to compress, resolution by resolution, without redundancy, and eases transmission or direct coding with the most suitable resolution to our display system.

Moreover, it is possible to arrange information in the code stream with an increasing quality. Figure 1 represents the unit schema of a JPEG2000 coder. As it appears, a typical algorithm of JPEG2000 coding is mainly divided into 5 modules: Color transform, discrete wavelets transform, quantification, entropy coding unit and rate allowance [5]

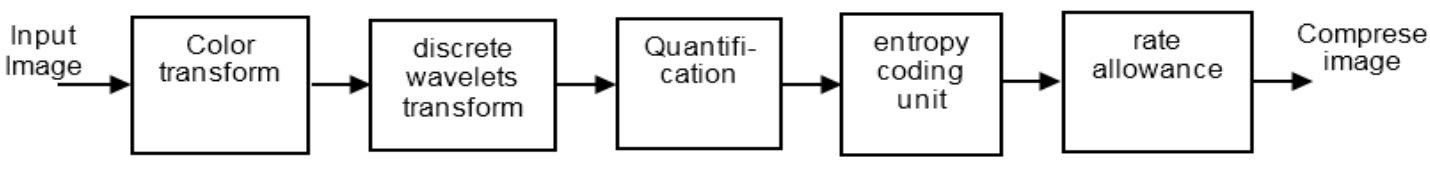

Figure 1. Typical Schema of a JPEG2000 Coder

\subsection{Wavelet Transform}

We can adapt wavelet transform in case of a discrete set. Such a technique is especially used with digital data with or without loss. Compression is achieved through successive approximations of initial information from the roughest to the finest. Therefore, we reduce information size by choosing a detail level [6].

Hence, we make a sampling of $s$ and $\tau$ on a dyadic scale. We have then:

$$
\psi_{m, n}[t]=s_{0}^{-m / 2} \psi\left(s_{0}^{-m} t-n \tau_{0}\right)
$$

Where $s_{0}$ and $\tau_{0}$ are constants.

We define discrete wavelet transform as follows:

$$
g[t]=\sum_{m \in \mathbb{Z}} \sum_{m \in \mathbb{Z}}\left\langle x, \psi_{m, n}\right\rangle \cdot \psi_{m, n}[t]
$$

\subsubsection{Extending Wavelet Transform to Bi-dimensional Signals}

Wavelet models can be generalized to every dimension $n>0$. In this research work, we make special emphasis on bi-dimensional cases to treat images [7-9].

Bi-dimensional signals are assumed measurable and having finite energy: $f(x, y) \in L^{2}\left(R^{2}\right)$.

Multi resolution analysis of $L^{2}\left(R^{2}\right)$ is obtained by defining it as a series of vector sub spaces ${ }_{V_{j}^{2}}$ of $L^{2}\left(R^{2}\right)$ We can define detail samples of $\mathrm{j}$ resolution as coefficients resulting from signal projection on ${ }^{W_{j}}$, orthogonal complement of $V_{j}$ and $V_{j-1}$. We can define an orthonormal basis of $W_{j}$ by translating and expanding three functions of $2 \mathrm{D}$ wavelet as follows: 


$$
\begin{aligned}
& \psi^{1}(x, y)=\phi(x) \psi(y) \\
& \psi^{2}(x, y)=\psi(x) \phi(y) \\
& \psi^{3}(x, y)=\psi(x) \psi(y)
\end{aligned}
$$

These functions are verified, if $\psi_{j, k, l}^{i}(x, y)=2^{-j} \psi^{i}\left(2^{-j} x-k, 2^{-j} y-l\right)$.

Hence $\left(\psi_{j, k, l}^{1}, \psi_{j, k, l}^{2}, \psi_{j, k, l}^{3}\right)_{(k, l) \in Z^{2}}$ is an orthonormal basis of $W_{j}$ and $\left(\psi_{j, k, l}^{1}, \psi_{j, k, l}^{2}, \psi_{j, k, l}^{3}\right)_{(j, k, l) \in Z^{3}}$ and is an orthonormal basis of $L^{2}\left(R^{2}\right)$.

\section{SPIHT CODING ALGORITHM (SET PARTITIONING IN HIERARCHICAL TREE)}

SPIHT algorithm (Figure 2) (Set Partitioning In Hierarchical Tree), were suggested by Saïd and Pearlman. It is based on the following concepts: progressive coding by bit plans and using hierarchical dependencies which are sustained by coefficients of a 2D breaking down pyramid. SPIHT algorithm puts forward a list of insignificant sets (LSP), a list of insignificant coefficient (LIS) and a list of significant coefficient (LIP). This algorithm uses a description pass of insignificant coefficients and a refining pass [2,3]

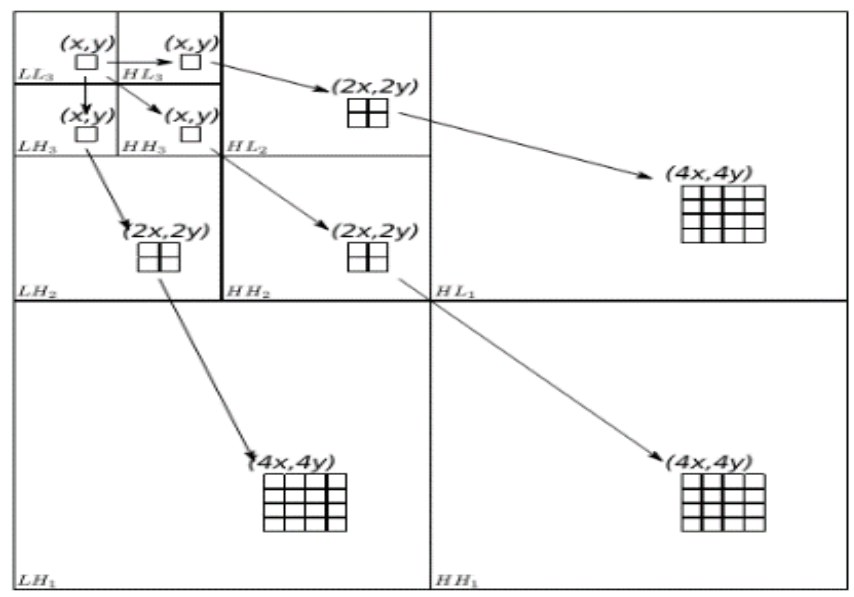

Figure 2. SPIHT Algorithm

\section{QUALITY EVALUATION PARAMETERS}

Measuring fidelity in compressing images is generally achieved by means of Mean Squaring Error MSE. Such a size (error) is defined by the mean square between the pixel $(i, j)$ of the original image $I(i, j)$, and the pixel $(i, j)$ of the reconstructed image $\hat{\imath}(i, j)_{i}$.

$$
M S E=\frac{1}{M \cdot N} \cdot \sum_{m=0}^{M-1} \sum_{n=0}^{N-1}[I(i, j)-\hat{I}(i, j)]^{2}
$$

The peak signal to noise ratio [10]

$$
P S N R=10 . \log _{10} \frac{\left(2^{R}-1\right)^{2}}{M S E}[d B]
$$

Afterwards, we evaluate a new paradigm to assess medical images quality. The similarity index compares brightness, contrast and structure between every vector pair, where structural similarity index (SSIM) between two signals $\mathrm{x}$ and $\mathrm{y}$ is expressed as follows: $\operatorname{SSIM}(x, y)=l(x, y) c(x, y) s(x, y)$.

Finally, for the purpose, we need a single global measure of overall image quality that is given by the formula:

$$
\operatorname{MSSIM}(I, \hat{I})=\frac{1}{M} \sum_{i=1}^{M} \operatorname{SSIM}\left(I_{i}, \hat{I}_{i}\right)
$$


$\mathrm{M}$ is the total number of local windows in the image. The values MSSIM exhibit greater consistency with the visual quality [11].

The Visual Information fidelity parameter (VIF) quantifies Shannon information that is shared between the reference and distorted images with respect to the information contained in the reference image itself [12].

VIF test is then evaluated as:

$$
\mathrm{VIF}=\frac{\sum_{\mathrm{j}}^{\mathrm{M}} \mathrm{I}\left(\mathrm{C}^{\mathrm{j}} ; \frac{\mathrm{j}}{\mathrm{j}}\right)}{\sum_{\mathrm{j}}^{\mathrm{M}} \mathrm{I}\left(\mathrm{C} ; \frac{\mathrm{j}}{\mathrm{j}} \mathrm{\frac { \textrm {j } } { \mathrm { j } }}\right)}
$$

Where, I $(\mathrm{X} ; \mathrm{Y} / \mathrm{Z})$ is the conditional mutual information between $\mathrm{X}$ and $\mathrm{Y}$, conditioned to $\mathrm{Z} ; \boldsymbol{S}^{j}$ is a realization of $S^{j}$ for a particular image, the index j runs through all sub-bands in the decomposed image [13, 14].

\section{RESULTS AND ANALYSIS}

\subsection{Method of Images Selection}

In this research work, we have made choices, among 300 images (100 natural images, 100 satellite images and 100 medical images), according to the following criteria:

a. Histogram: It enables a great amount of information in terms of grey levels (intensity) distribution [15].

b. Entropy: It informs us about information quantity conveyed in the image [16].

c. Standard deviation: It is the intensity dispersion measurement regarding its average [15].

This research aim is to collect a set of 20 test images for every type (medical, satellite and natural), in order to check compression algorithms efficiency. We adopted a selection algorithm based on statistics achieved according to image evaluation parameters determined above.

\subsection{Selection Algorithm for the Test Images}

Selection algorithm for the test images is summarized in the flow chart of the Figure 3, It goes, after choosing $\mathrm{N}$ images $(\mathrm{N}=100$ images in this paper) and $\mathrm{K}$ bit rates $\mathrm{Rc}$ for every image (in this paper, we took $\mathrm{Rc}=0.25,0.50, \ldots, 3.00$, where $\mathrm{K}=12)$, through variances calculation [15] $\sigma_{-}(\mathrm{PSNR} \mathrm{i})^{\wedge} 2, \sigma_{-}(\mathrm{MSSIM} \mathrm{i})^{\wedge} 2$ et $\sigma \_\left(V I F \_i\right) \wedge 2$ evaluation parameters by

$$
\begin{aligned}
& \sigma_{P_{S N R}}^{2}=\frac{1}{K} \sum_{j=1}^{K}\left(\operatorname{PSNR}_{i, j}-m_{P_{S N R}}\right)^{2} \\
& \sigma_{\text {MSSIM }_{i}}^{2}=\frac{1}{\mathrm{~K}} \sum_{\mathrm{j}=1}^{\mathrm{K}}\left(\operatorname{MSSIM}_{\mathrm{i}, \mathrm{j}}-\mathrm{m}_{\mathrm{MSSIM}_{\mathrm{j}}}\right)^{2} \\
& \sigma_{\mathrm{VIF}_{\mathrm{i}}}^{2}=\frac{1}{\mathrm{~K}} \sum_{\mathrm{j}=1}^{\mathrm{K}}\left(\mathrm{VIF}_{\mathrm{i}, \mathrm{j}}-\mathrm{m}_{\mathrm{VIF}_{\mathrm{j}}}\right)^{2}
\end{aligned}
$$

With, PSNR_(i,j), MSSIM_(i,j) and VIF_(i,j) are values of evaluation images parameters $i=1 \ldots N$ and bit rates $R c \_j, j=1 \ldots \bar{K} . m_{-}\left(P S N R \_\bar{j}\right), m_{-}\left(M S S I M \_j\right)$ et $m_{-}\left(V I F \_j\right)$ are evaluation parameters averages for $N$ images, and every $R c_{-} \mathrm{j}$, calculated as follows:

$$
\begin{aligned}
& \mathrm{m}_{\mathrm{PSNR}_{\mathrm{j}}}=\frac{1}{\mathrm{~N}} \sum_{\mathrm{i}=1}^{\mathrm{N}} \operatorname{PSNR}_{\mathrm{i}, \mathrm{j}} \\
& \mathrm{m}_{\mathrm{MSSIM}_{\mathrm{j}}}=\frac{1}{\mathrm{~N}} \sum_{\mathrm{i}=1}^{\mathrm{N}} \operatorname{MSSIM}_{\mathrm{i}, \mathrm{j}} \\
& \mathrm{m}_{\mathrm{VIF}_{\mathrm{j}}}=\frac{1}{\mathrm{~N}} \sum_{\mathrm{i}=1}^{\mathrm{N}} \mathrm{VIF}_{\mathrm{i}, \mathrm{j}}
\end{aligned}
$$

The last step of the algorithm consists of selecting $\mathrm{M}$ images among $\mathrm{N}$ previous choices, based on variances thresholds choices $S_{\mathrm{PSNR}}, \mathrm{S}_{\mathrm{MSSIM}}$ et $\mathrm{S}_{\mathrm{VIF}}$ enabling to save only the images $\mathrm{i}=1 \ldots \mathrm{N}$ according to the following rules:

$\sigma_{\mathrm{PSNR}_{\mathrm{i}}}^{2} \leq \mathrm{S}_{\mathrm{PSNR}}, \sigma_{\mathrm{MSSIM}_{\mathrm{i}}}^{2} \leq \mathrm{S}_{\mathrm{MSSIM}}$ et $\sigma_{\mathrm{VIF}_{\mathrm{i}}}^{2} \leq \mathrm{S}_{\mathrm{VIF}}$ 


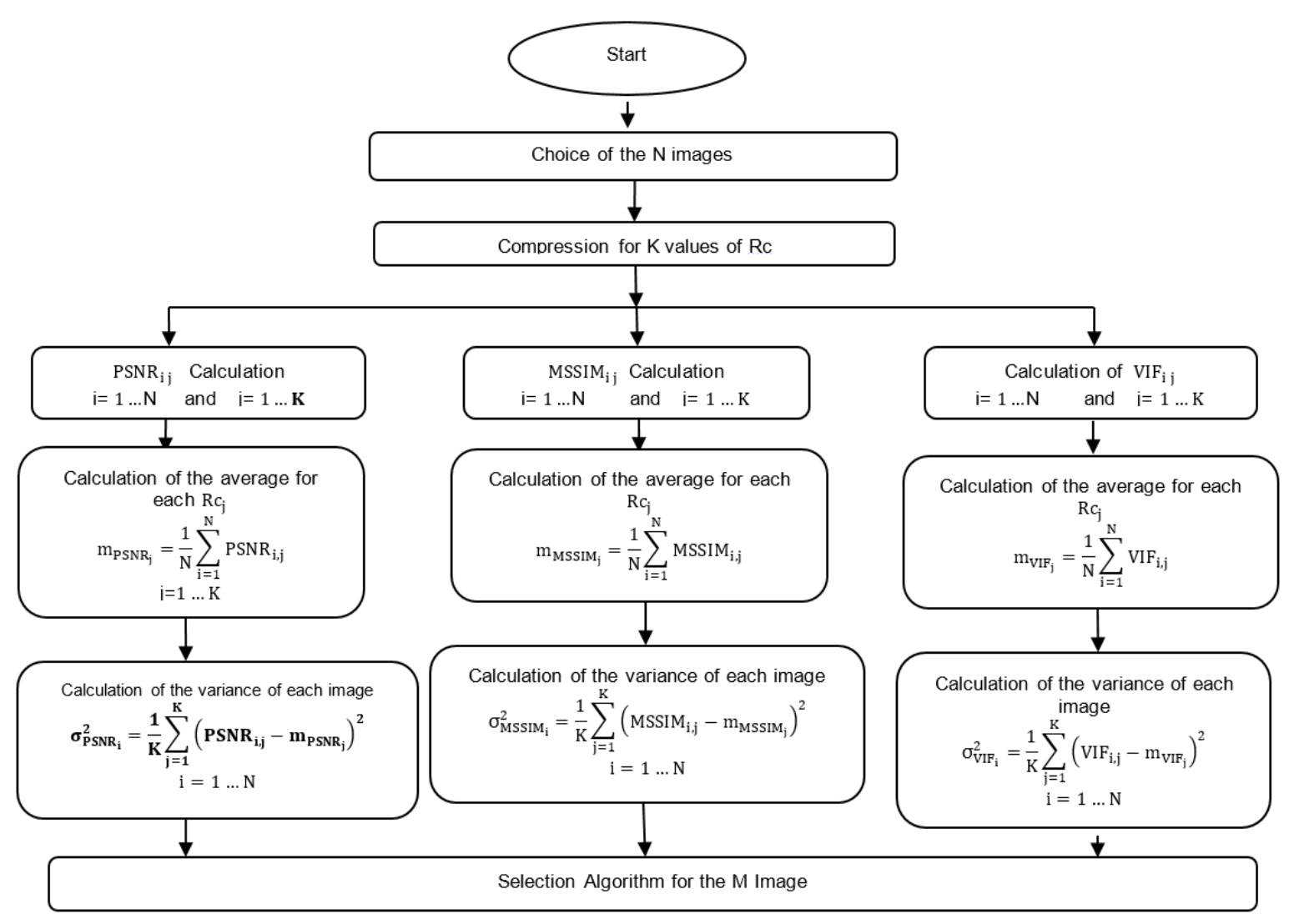

Figure 3. Chart of selection algorithm for test images

In order to give an example of the execution of the suggested algorithm, we chose $\mathrm{N}=5$ images $(1$, $9,16,31,22$ ) of size $512 \times 512$ in the grayscale (coded on 8bpp) represented in figure 4 . We calculated three evaluation parameters PSNR, MSSIM, VIF in terms of bit rates $\mathrm{Rc}=0.25,0.50, \ldots, 3.00$ ( $\mathrm{K}=12 \mathrm{Rc}$ values), by adopting DWT/SPIHT as compression algorithm.

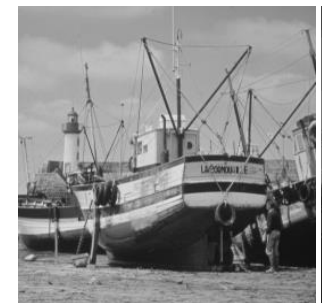

Boat (1)

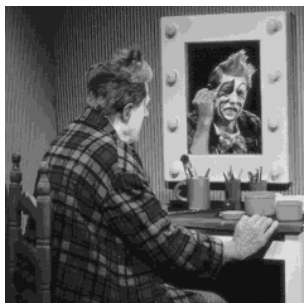

Clown (9)

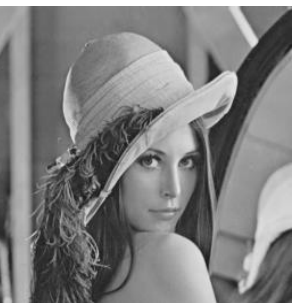

Lena (16)

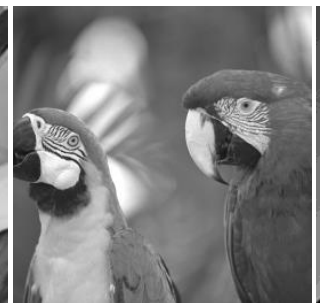

Bird (31)

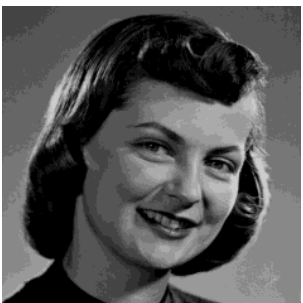

Woman (22)

Figure 4. Selected Images

Steps of averages calculation and variances of evaluation parameters are summarized in the Tables 1,2 and 3. According to average variances column as it appears in the tables, the selection algorithm of test images accepts as values of variances thresholds

$\llbracket 0.2385<\mathrm{S} \rrbracket$ PSNR $<2.1170,0.0000765<\mathrm{S} \_\mathrm{MSSIM}<0.0002874$ and $\llbracket 0.0002507<\mathrm{S} \rrbracket \_\mathrm{VIF}<0.0003864$ for $\mathrm{M}=1$ image (in this case the test image is « $16 »)$. In the case where $\mathrm{M}=2$, thresholds are as follows:

$2.1170<$ S_PSNR $<3.8181$, 『0.0002874 $<$ S \_MSSIM $<0.0003713$ and $0.0003864<$ S_VIF $<0.0008309$, and test images are « $16 »$ et $« 9 »$. 
Table 1. PSNR Variation

\begin{tabular}{|c|c|c|c|c|c|c|c|c|c|c|c|c|c|}
\hline & 0.25 & 0.50 & 0.75 & 1.00 & 1.25 & 1.50 & 1.75 & 2.00 & 2.25 & 2.50 & 2.75 & 3.00 & \\
\hline 1 & 26.85 & 31.08 & 32.95 & 34.96 & 35.83 & 36.87 & 38.29 & 39.28 & 39.86 & 40.80 & 42.14 & 43.95 & \multirow{6}{*}{$\begin{array}{l}\text { Average } \\
\text { Variance }\end{array}$} \\
\hline 9 & 27.83 & 31.86 & 34.14 & 36.60 & 38.01 & 40.20 & 41.23 & 42.29 & 43.75 & 45.09 & 45.92 & 46.55 & \\
\hline 16 & 30.49 & 34.68 & $\begin{array}{l}34.14 \\
37.08\end{array}$ & 38.66 & $\begin{array}{l}50.01 \\
39.96\end{array}$ & 40.69 & 41.62 & 42.99 & 44.09 & 45.05 & 45.65 & 46.51 & \\
\hline 31 & 31.81 & 37.39 & 39.82 & 41.95 & 43.09 & 44.54 & 45.42 & 46.07 & 47.02 & 48.16 & 49.72 & 50.77 & \\
\hline 22 & 31.84 & 36.36 & 38.59 & 40.64 & 41.70 & 42.72 & 44.26 & 45.26 & 45.84 & 46.75 & 47.94 & 49.63 & \\
\hline $\begin{array}{l}\text { Average } \\
\text { PSNR }\end{array}$ & 29.76 & 34.27 & 36.52 & 38.56 & 39.72 & 41.01 & 42.17 & 43.18 & 44.11 & 45.1691 & 46.28 & 47.48 & \\
\hline 1 & 08.48 & 10.21 & 12.72 & 13.02 & 15.14 & 17.12 & 15.03 & 15.21 & 18.08 & 19.11 & 17.08 & 12.49 & 14.47 \\
\hline 9 & 03.73 & 05.81 & 05.66 & 03.84 & 02.92 & 00.65 & 00.87 & 00.78 & 00.13 & 00.01 & 00.13 & 00.87 & 02.12 \\
\hline 16 & 00.52 & 00.17 & 00.32 & 00.01 & 00.06 & 00.10 & 00.30 & 00.04 & 00.00 & 00.01 & 00.39 & 00.95 & 00.24 \\
\hline 31 & 04.18 & 09.69 & 10.93 & 11.50 & 11.36 & 12.53 & 10.61 & 08.38 & 08.47 & 08.92 & 11.86 & 10.81 & 09.94 \\
\hline 22 & 04.31 & 04.35 & 04.31 & 04.32 & 03.94 & 02.95 & 04.40 & 04.33 & 02.99 & 02.51 & 02.77 & 04.63 & 03.82 \\
\hline
\end{tabular}

Table 2. MSSIM Variation

\begin{tabular}{|c|c|c|c|c|c|c|c|c|c|c|c|c|c|}
\hline & 0.25 & 0.50 & 0.75 & 1.00 & 1.25 & 1.50 & 1.75 & 2.00 & 2.25 & 2.50 & 2.75 & 3.00 & \\
\hline 1 & 0.698 & 0.821 & 0.863 & 0.896 & 0.908 & 0.925 & 0.946 & 0.956 & 0.960 & 0.966 & 0.976 & 0.984 & \multirow{6}{*}{$\begin{array}{l}\text { Average } \\
\text { Variance }\end{array}$} \\
\hline 9 & 0.746 & 0.880 & 0.925 & 0.949 & 0.960 & 0.973 & 0.976 & 0.980 & 0.985 & 0.988 & 0.990 & 0.991 & \\
\hline 16 & 0.826 & 0.896 & 0.922 & 0.939 & 0.949 & 0.955 & 0.962 & 0.971 & 0.978 & 0.982 & 0.983 & 0.986 & \\
\hline 31 & 0.871 & 0.932 & 0.951 & 0.963 & 0.969 & 0.977 & 0.980 & 0.982 & 0.985 & 0.989 & 0.992 & 0.994 & \\
\hline 22 & 0.851 & 0.918 & 0.939 & 0.955 & 0.960 & 0.967 & 0.976 & 0.980 & 0.982 & 0.985 & 0.988 & 0.992 & \\
\hline $\begin{array}{l}\text { Average } \\
\text { MSSIM }\end{array}$ & 0.799 & 0.889 & 0.920 & 0.940 & 0.950 & 0.959 & 0.968 & 0.974 & 0.978 & 0.982 & 0.986 & 0.989 & \\
\hline 1 & 0.0101 & 0.0047 & 0.0033 & 0.0019 & 0.0017 & 0.0012 & 0.0005 & 0.0003 & 0.0003 & 0.0002 & 0.0001 & 0.0000 & 0.0020 \\
\hline 9 & 0.0028 & 0.0001 & 0.0000 & 0.0001 & 0.0001 & 0.0002 & 0.0001 & 0.0000 & 0.0001 & 0.0000 & 0.0000 & 0.0000 & 0.0003 \\
\hline 16 & 0.0008 & 0.0001 & 0.0000 & 0.0000 & 0.0000 & 0.0000 & 0.0000 & 0.0000 & 0.0000 & 0.0000 & 0.0000 & 0.0000 & 0.0001 \\
\hline 31 & 0.0053 & 0.0018 & 0.0010 & 0.0005 & 0.0004 & 0.0003 & 0.0001 & 0.0001 & 0.0001 & 0.0000 & 0.0000 & 0.0000 & 0.0008 \\
\hline 22 & 0.0028 & 0.0008 & 0.0004 & 0.0002 & 0.0001 & 0.0001 & 0.0001 & 0.0000 & 0.0000 & 0.0000 & 0.0000 & 0.0000 & 0.0004 \\
\hline
\end{tabular}

Table 3. VIF Variation

\begin{tabular}{|c|c|c|c|c|c|c|c|c|c|c|c|c|c|}
\hline & 0.25 & 0.50 & 0.75 & 1.00 & 1.25 & 1.50 & 1.75 & 2.00 & 2.25 & 2.50 & 2.75 & 3.00 & \\
\hline 1 & 0.265 & 0.424 & 0.501 & 0.569 & 0.604 & 0.641 & 0.689 & 0.722 & 0.740 & 0.771 & 0.810 & 0.853 & \multirow{6}{*}{$\begin{array}{l}\text { Average } \\
\text { Variance }\end{array}$} \\
\hline 9 & 0.312 & 0.479 & 0.568 & 0.645 & 0.692 & 0.753 & 0.781 & 0.811 & 0.847 & 0.876 & 0.891 & 0.902 & \\
\hline 16 & 0.386 & 0.540 & 0.614 & 0.664 & 0.706 & 0.731 & 0.763 & 0.805 & 0.835 & 0.859 & 0.871 & 0.891 & \\
\hline 31 & 0.405 & 0.572 & 0.653 & 0.717 & 0.757 & 0.804 & 0.830 & 0.846 & 0.871 & 0.896 & 0.924 & 0.940 & \\
\hline 22 & 0.396 & 0.543 & 0.619 & 0.687 & 0.724 & 0.760 & 0.809 & 0.838 & 0.851 & 0.874 & 0.899 & 0.928 & \\
\hline $\begin{array}{l}\text { Average } \\
\text { VIF }\end{array}$ & 0.353 & 0.512 & 0.591 & 0.656 & 0.697 & 0.738 & 0.774 & 0.804 & 0.829 & 0.855 & 0.879 & 0.903 & \\
\hline 1 & 0.0076 & 0.0077 & 0.0082 & 0.0076 & 0.0086 & 0.0095 & 0.0073 & 0.0068 & 0.0079 & 0.0071 & 0.0048 & 0.0025 & 0.0071 \\
\hline 9 & 0.0017 & 0.0010 & 0.0005 & 0.0001 & 0.0000 & 0.0002 & 0.0001 & 0.0000 & 0.0003 & 0.0004 & 0.0002 & 0.0000 & 0.0004 \\
\hline 16 & 0.0011 & 0.0008 & 0.0005 & 0.0001 & 0.0001 & 0.0000 & 0.0001 & 0.0000 & 0.0000 & 0.0000 & 0.0001 & 0.0001 & 0.0003 \\
\hline 31 & 0.0027 & 0.0036 & 0.0039 & 0.0037 & 0.0037 & 0.0043 & 0.0030 & 0.0018 & 0.0018 & 0.0017 & 0.0020 & 0.0014 & 0.0028 \\
\hline 22 & 0.0019 & 0.0010 & 0.0008 & 0.0009 & 0.0007 & 0.0005 & 0.0012 & 0.0011 & 0.0005 & 0.0003 & 0.0004 & 0.0006 & 0.0008 \\
\hline
\end{tabular}

To expand the application of the selection algorithm to $M=20$ images, we chose $N=100$ images (100 natural images, 100 satellite images and 100 medical images) of size $512 \times 512$ in the grayscale (coded on $8 \mathrm{bpp}$ ). We adopted $\mathrm{K}=12$ values of the bit rate $\mathrm{Rc}=0.25,0.50, \ldots, 3.00$ and by using two wavelet-based compression algorithms and JPEG2000.

Selected images compressed by CDF9/7 algorithm with lifting structure and coupled with SPIHT encoder, gave the evaluation parameters (PSNR, MSSIM and VIF) values represented in Figures 5, 6, and 7. The variation intervals of the PSNR are as follows:

a. PSNR of 100 medical images varies between $25 \mathrm{~dB}$ and $48 \mathrm{~dB}$ for the bit rates $\mathrm{Rc}=0.25 \mathrm{bpp}$, and between $45 \mathrm{~dB}$ and $68 \mathrm{~dB}$ for the bit rates $\mathrm{Rc}=3 \mathrm{bpp}$.

b. PSNR of 100 natural images varies between $20 \mathrm{~dB}$ and $42 \mathrm{~dB}$ for the bit rates $\mathrm{Rc}=0.25 \mathrm{bpp}$, and between $35 \mathrm{~dB}$ and $62 \mathrm{~dB}$ for the bit rates $\mathrm{Rc}=3 \mathrm{bpp}$.

c. PSNR of 100 satellite images varies between $19 \mathrm{~dB}$ and $35 \mathrm{~dB}$ for the bit rates $\mathrm{Rc}=0.25 \mathrm{bpp}$, and between $32 \mathrm{~dB}$ and $52 \mathrm{~dB}$ for the bit rates $\mathrm{Rc}=3 \mathrm{bpp}$ 


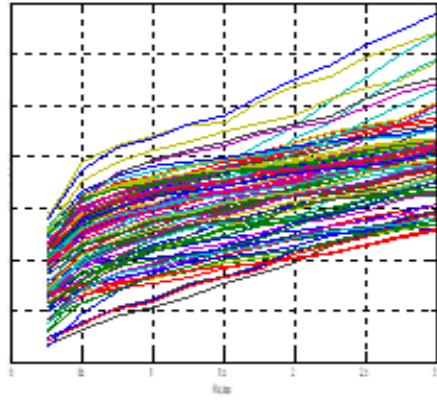

PNSR

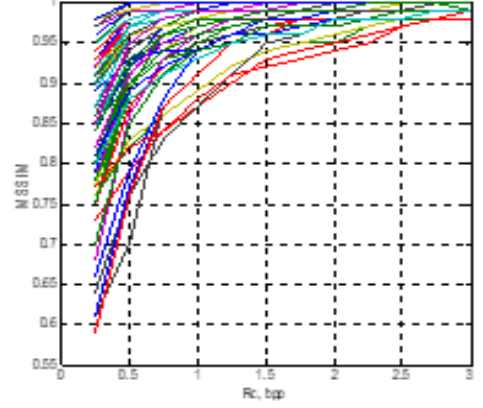

MSSIM

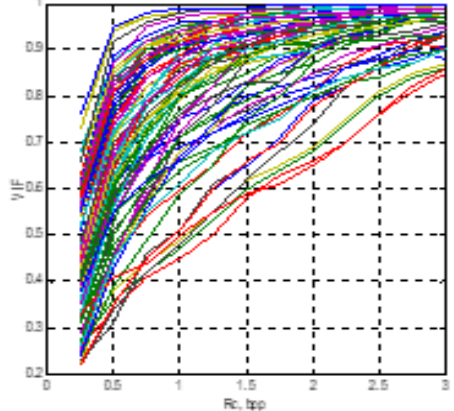

VIF

Figure 5. PSNR, MSSIM and VIF variation of 100 medical images
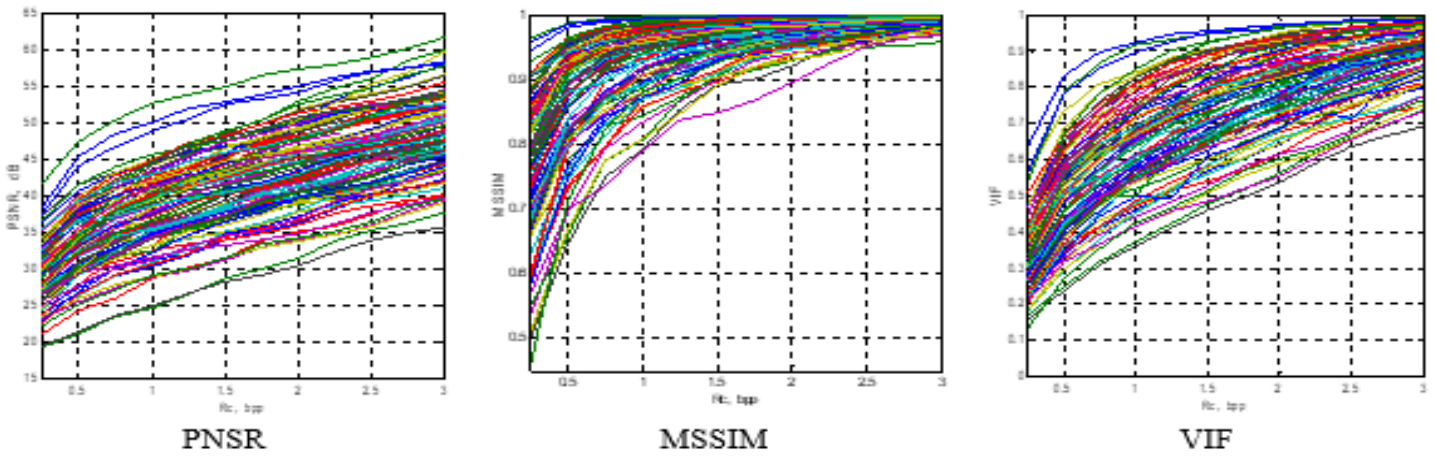

Figure 6. PSNR, MSSIM and VIF variation of 100 natural images

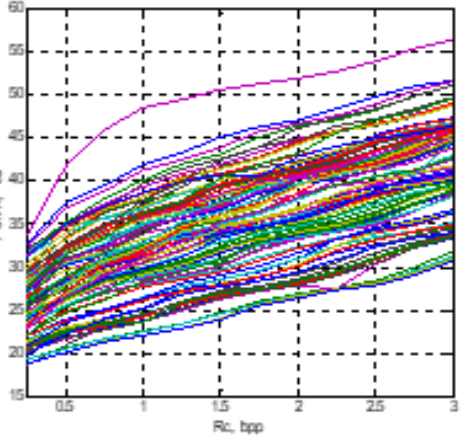

PNSR

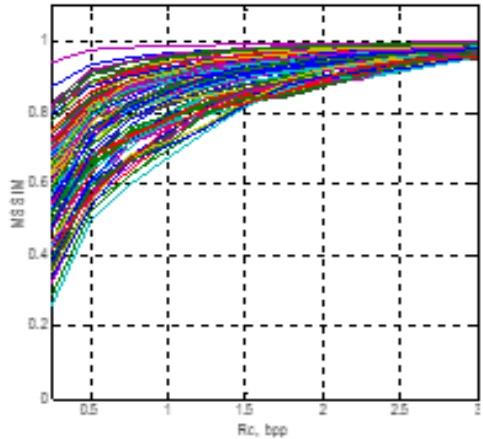

MSSIM

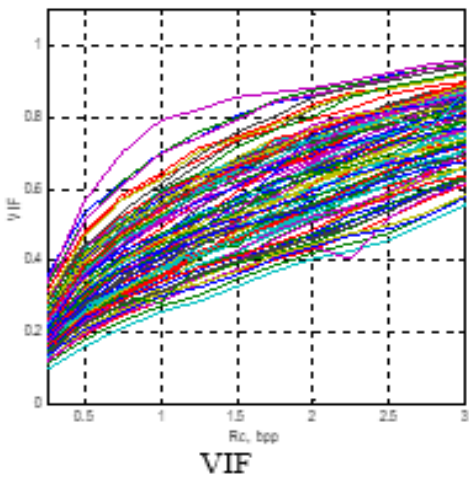

Figure 7. PSNR, MSSIM and VIF variation of 100 satellite images

Table 4 represents the resulting test Images by the selection algorithm, according to image types (medical, natural and satellite). In order to obtain the 20 test images, the applied thresholds on satellite images, enabled to choose 32 images from PSNR, 35 images from MSSIM and 38 images from VIF. For 20 natural test images, the selection algorithm selected 29 images from PSNR, 24 images from MSSIM and 42 images from VIF. The selected images for medicla images are, respectively, 21, 37 and 43. The test images are represented in Figures 8, 9, and 10. 
Table 4. Resulting Test Images by the Selection Algorithm

\begin{tabular}{|c|c|c|c|c|c|c|c|c|}
\hline \multicolumn{3}{|c|}{ Image Natural } & \multicolumn{3}{|c|}{ Image Medical } & \multicolumn{3}{|c|}{ Image Satellite } \\
\hline PSNR & MSSIM & VIF & PSNR & MSSIM & VIF & PSNR & MSSIM & VIF \\
\hline 9 & 9 & 9 & 5 & 1 & 1 & 5 & 1 & 1 \\
\hline 16 & 15 & 15 & 11 & 5 & 5 & 6 & 5 & 3 \\
\hline 21 & 16 & 16 & 14 & 8 & 11 & 10 & 6 & 5 \\
\hline 22 & 21 & 17 & 24 & 11 & 14 & 12 & 7 & 6 \\
\hline 26 & 26 & 21 & 25 & 14 & 15 & 15 & 8 & 7 \\
\hline 30 & 30 & 22 & 26 & 18 & 16 & 19 & 10 & 8 \\
\hline 33 & 33 & 26 & 32 & 22 & 18 & 23 & 12 & 9 \\
\hline 39 & 37 & 30 & 37 & 24 & 19 & 24 & 15 & 10 \\
\hline 40 & 39 & 31 & 41 & 25 & 23 & 25 & 19 & 12 \\
\hline 46 & 46 & 32 & 49 & 26 & 24 & 27 & 21 & 14 \\
\hline 48 & 54 & 33 & 57 & 29 & 25 & 28 & 24 & 15 \\
\hline 49 & 57 & 35 & 61 & 32 & 26 & 30 & 25 & 19 \\
\hline 53 & 59 & 37 & 66 & 33 & 27 & 31 & 27 & 21 \\
\hline 59 & 60 & 39 & 67 & 34 & 32 & 32 & 28 & 22 \\
\hline 60 & 61 & 46 & 73 & 37 & 33 & 34 & 34 & 24 \\
\hline 61 & 63 & 49 & 76 & 40 & 34 & 36 & 40 & 25 \\
\hline 63 & 67 & 54 & 78 & 41 & 37 & 38 & 41 & 30 \\
\hline 67 & 69 & 56 & 80 & 44 & 41 & 40 & 42 & 34 \\
\hline 69 & 73 & 57 & 82 & 49 & 45 & 43 & 43 & 35 \\
\hline 73 & 77 & 59 & 88 & 57 & 49 & 46 & 46 & 37 \\
\hline 77 & 84 & 60 & 91 & 60 & 57 & 48 & 47 & 40 \\
\hline 80 & 85 & 61 & & 61 & 58 & 74 & 48 & 41 \\
\hline 81 & 88 & 63 & & 66 & 60 & 76 & 71 & 42 \\
\hline 83 & 93 & 66 & & 67 & 61 & 78 & 74 & 43 \\
\hline 84 & & 67 & & 73 & 63 & 84 & 76 & 45 \\
\hline 85 & & 68 & & 74 & 66 & 85 & 78 & 46 \\
\hline 88 & & 69 & & 76 & 67 & 86 & 79 & 47 \\
\hline 93 & & 71 & & 77 & 72 & 93 & 84 & 48 \\
\hline \multirow[t]{15}{*}{96} & & 73 & & 78 & 73 & 94 & 85 & 71 \\
\hline & & 74 & & 80 & 74 & 95 & 86 & 74 \\
\hline & & 77 & & 81 & 76 & 96 & 88 & 76 \\
\hline & & 80 & & 82 & 77 & 97 & 93 & 78 \\
\hline & & 81 & & 83 & 78 & & 95 & 79 \\
\hline & & 83 & & 84 & 80 & & 96 & 85 \\
\hline & & 84 & & 91 & 81 & & 99 & 86 \\
\hline & & 85 & & 96 & 82 & & & 93 \\
\hline & & 88 & & 100 & 83 & & & 96 \\
\hline & & 93 & & & 84 & & & 99 \\
\hline & & 95 & & & 85 & & & \\
\hline & & 96 & & & 88 & & & \\
\hline & & 97 & & & 91 & & & \\
\hline & & 98 & & & 96 & & & \\
\hline & & & & & 100 & & & \\
\hline
\end{tabular}

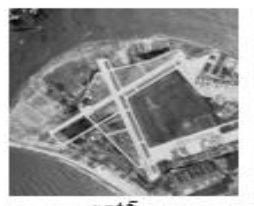

sat5

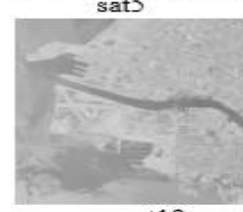

sat 19

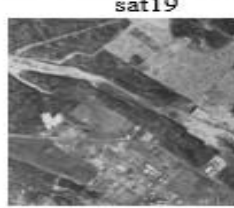

sat43

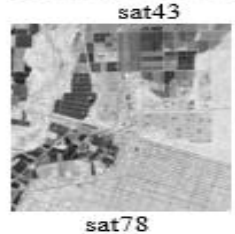

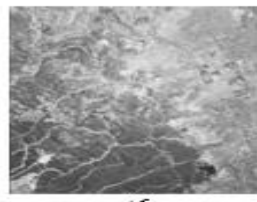

sat6

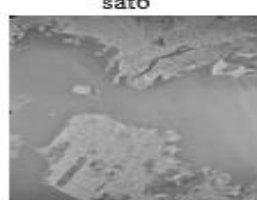

sat 24
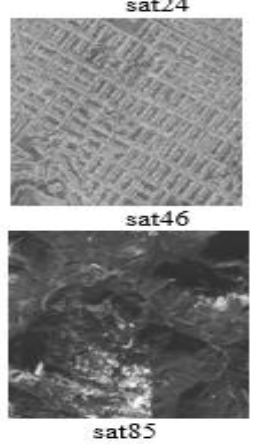

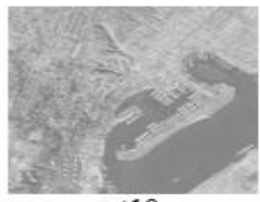

sat 10

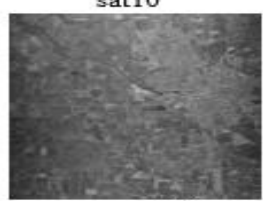

sat 25

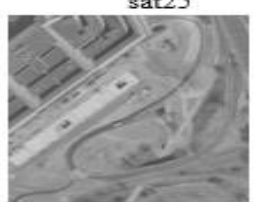

sat 48

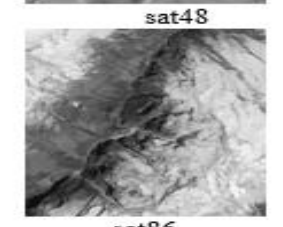

sat86

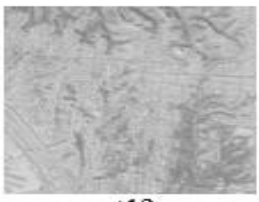

sat12

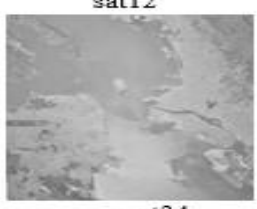

sat34
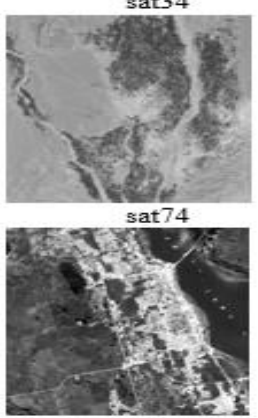

sat93

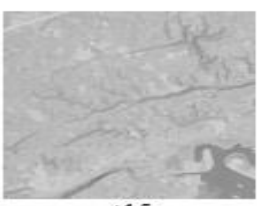

sat15

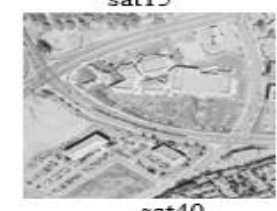

sat 40
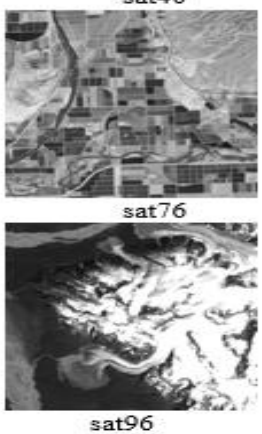

Figure 8 . The 20 selected satellite test images 


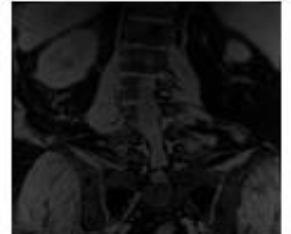

med5
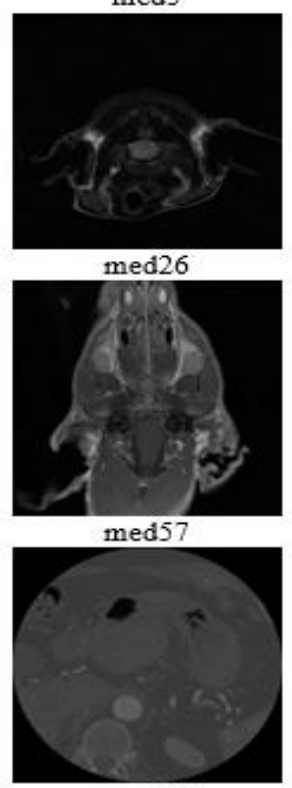

$\operatorname{med} 76$

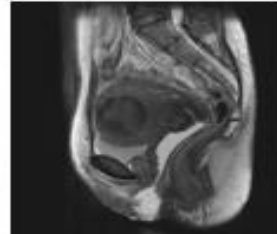

med11

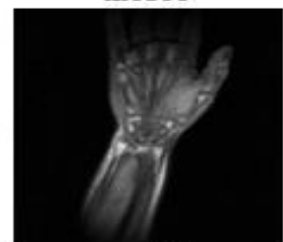

$\operatorname{med} 32$

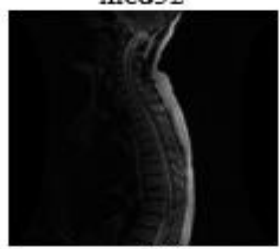

med61

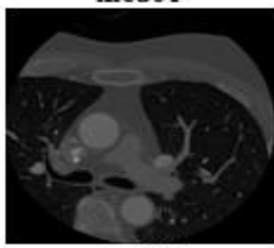

$\operatorname{med} 78$

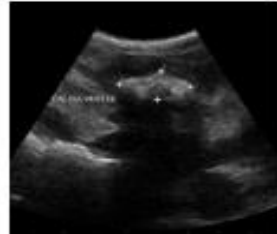

$\operatorname{med} 14$

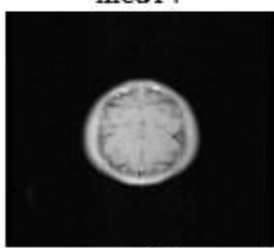

$\operatorname{med} 37$

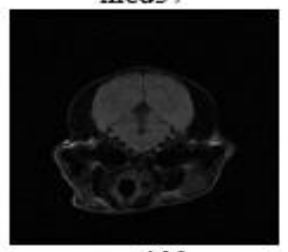

med66

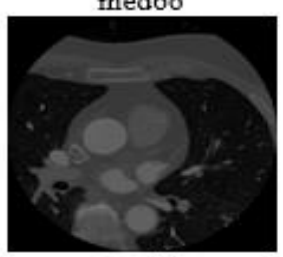

$\operatorname{med} 80$

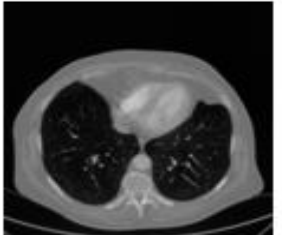

$\operatorname{med} 24$

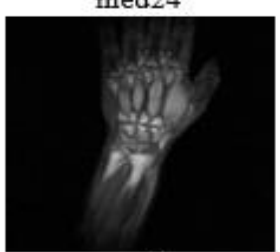

med41

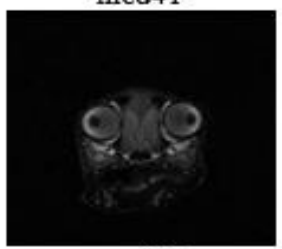

$\operatorname{med} 67$

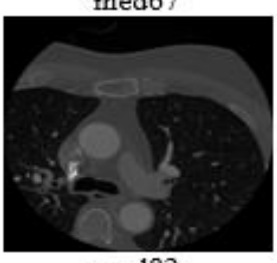

$\operatorname{med} 82$

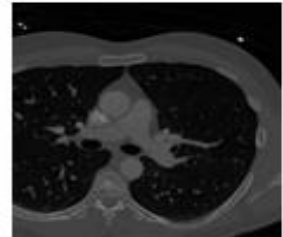

$\operatorname{med} 25$

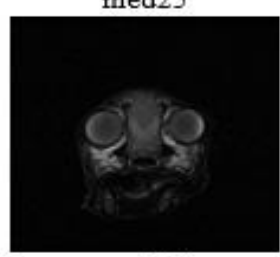

med49

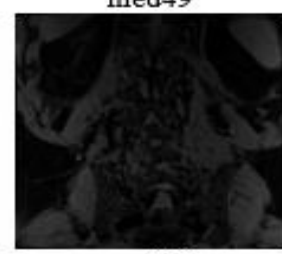

$\operatorname{med} 73$

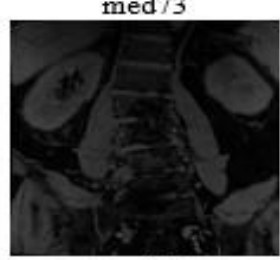

med 91

Figure 9. The 20 selected medical test images

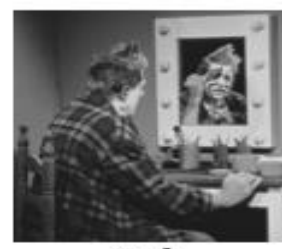

nat9

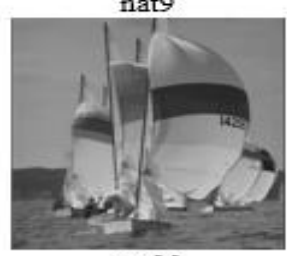

nat 30

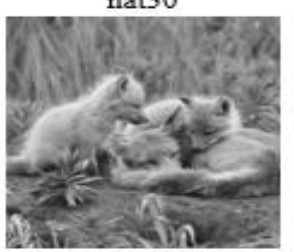

nat59

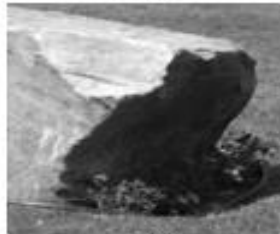

nat 73

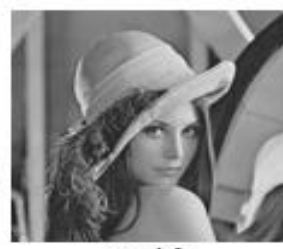

nat16

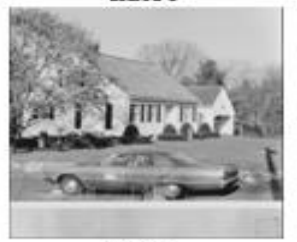

nat 33

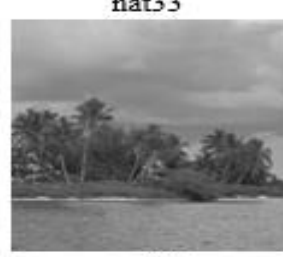

nat 60

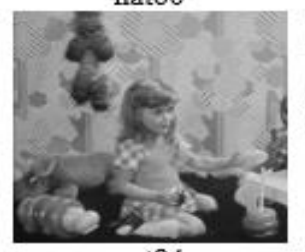

nat84

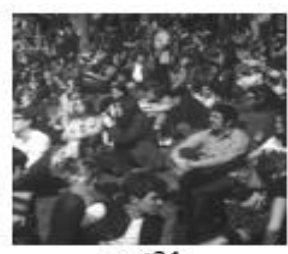

nat21
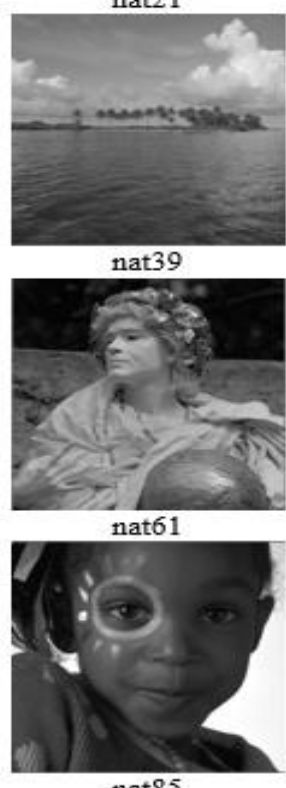

nat85

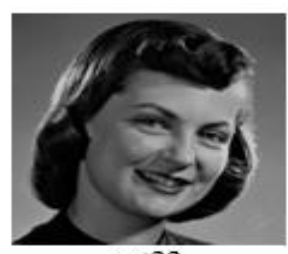

nat 22

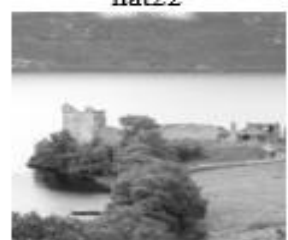

nat46

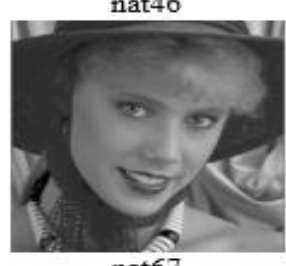

nat67

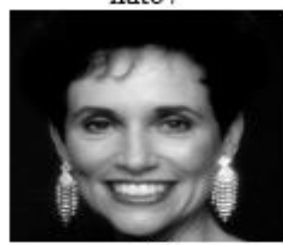

nat88
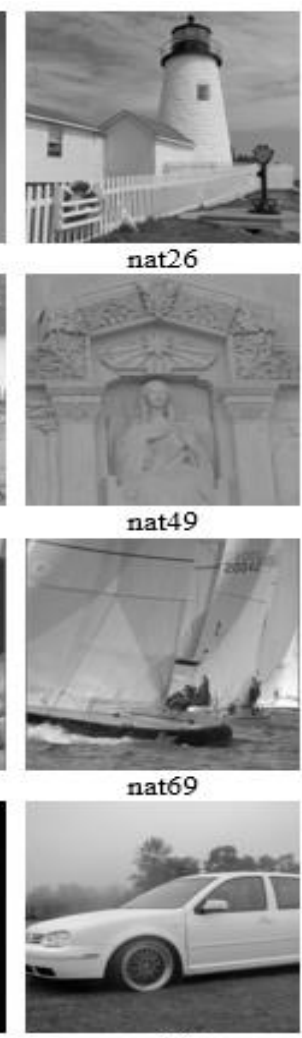

nat93

Figure 10. The 20 selected natural test images 
The evaluation parameters curves of the 20 test images per image type are represented in figures 11 , 12 and 13. It can be observed that the loss in PSNR, MSSIM and VIF values is nearly constant whatever is the value of Rc for the three image types (medical, natural and satellite).
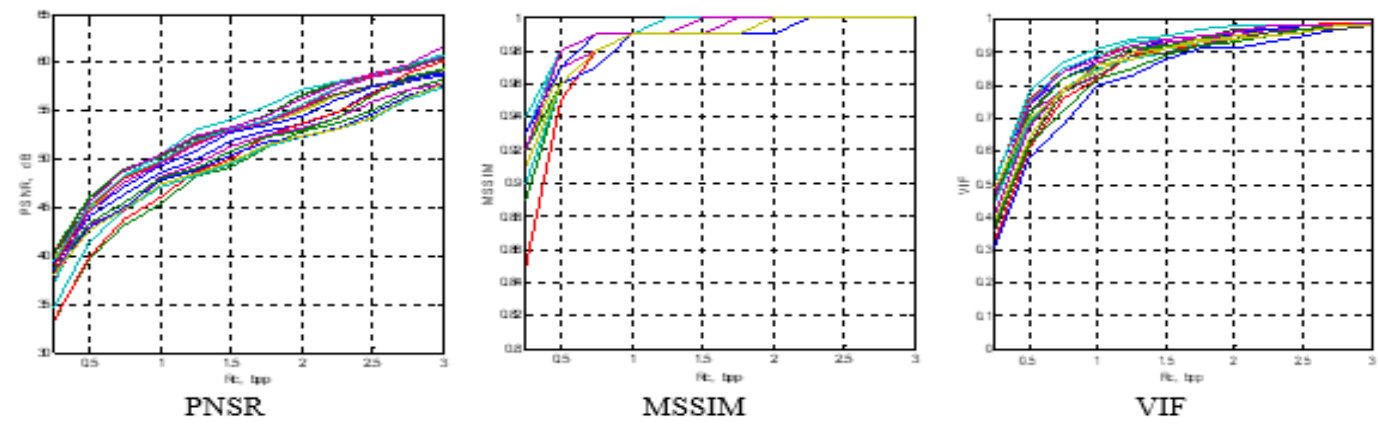

Figure 11. PSNR, MSSIM and VIF variations of the 20 selected medical test images using DWT+SPIHT
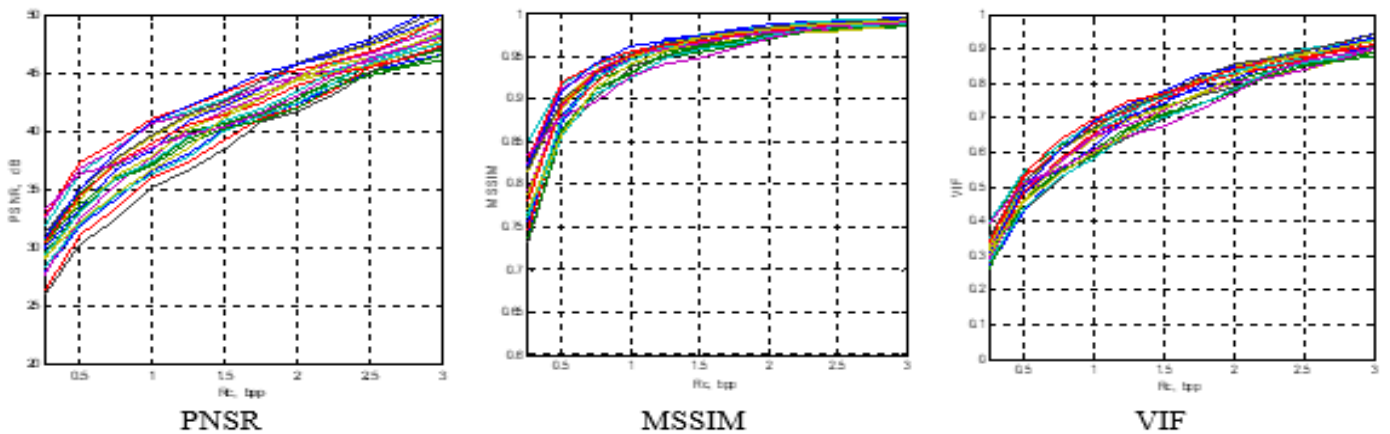

Figure 12. PSNR, MSSIM and VIF variations of the 20 selected natural test images using DWT+SPIHT
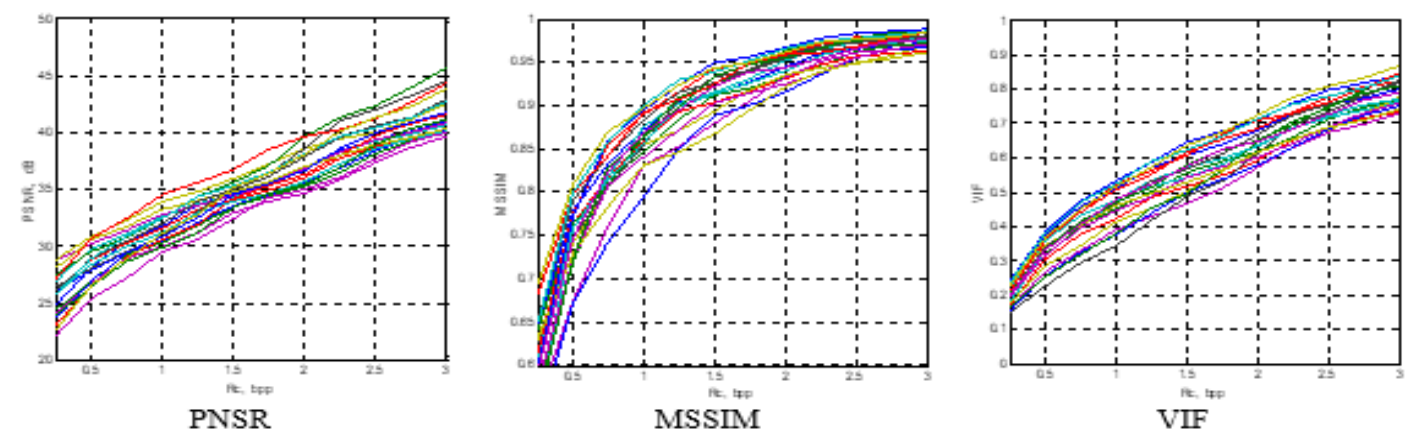

Figure 13. PSNR, MSSIM and VIF variations of the 20 selected satellite test images using DWT+SPIHT

The same prcedure was applied using JPEG 2000 standard as compression algorithm. The evaluation parameters variations are illustrated in Figures 14, 15 and 16. The variation intervals of the PSNR are as follows:

a. PSNR for 100 medical images varies between $26 \mathrm{~dB}$ and $58 \mathrm{~dB}$ for a bit rates of $\mathrm{Rc}=0.25 \mathrm{bpp}$, and between $47 \mathrm{~dB}$ and $60 \mathrm{~dB}$ for a bit rates of $\mathrm{Rc}=3 \mathrm{bpp}$.

b. PSNR for 100 natural images varies between $21 \mathrm{~dB}$ and $47 \mathrm{~dB}$ for a bit rates of $\mathrm{Rc}=0.25 \mathrm{bpp}$, and between $39 \mathrm{~dB}$ and $57 \mathrm{~dB}$ for a bit rates of $\mathrm{Rc}=3 \mathrm{bpp}$.

c. PSNR for 100 satellite images varies between $19 \mathrm{~dB}$ and $40 \mathrm{~dB}$ for a bit rates of $\mathrm{Rc}=0.25 \mathrm{bpp}$, and between $33 \mathrm{~dB}$ and $53 \mathrm{~dB}$ for a bit rates of $\mathrm{Rc}=3 \mathrm{bpp}$. 


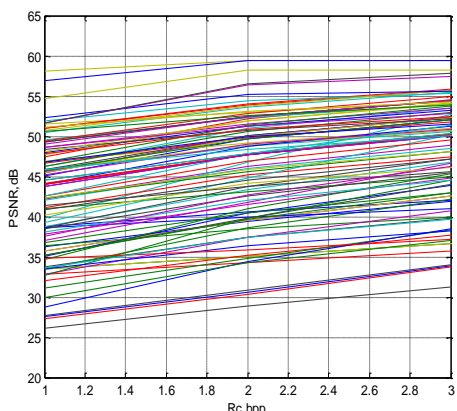

PSNR

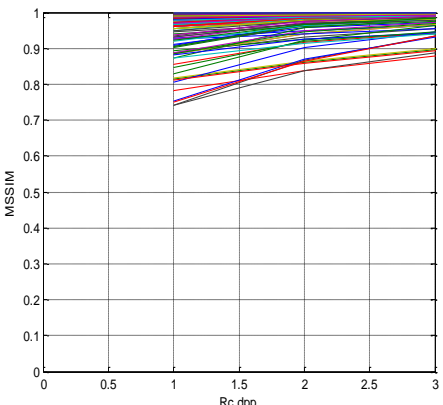

MSSIM

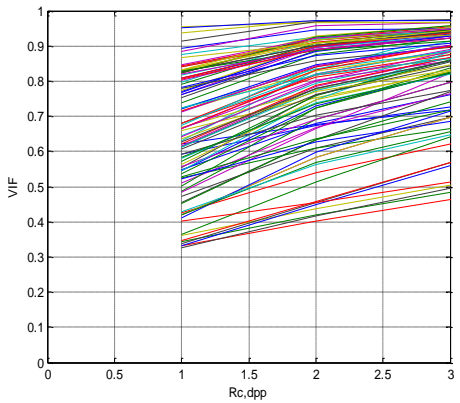

VIF

Figure 14. The variations of PSNR, MSSIM and VIF of 100 medical images

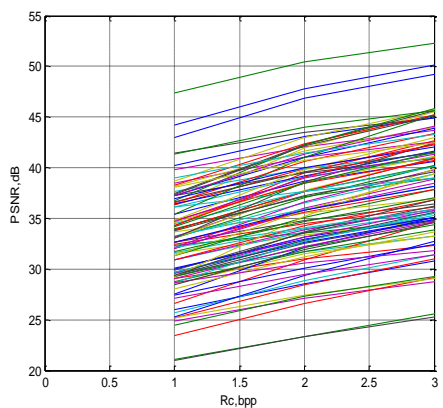

PSNR

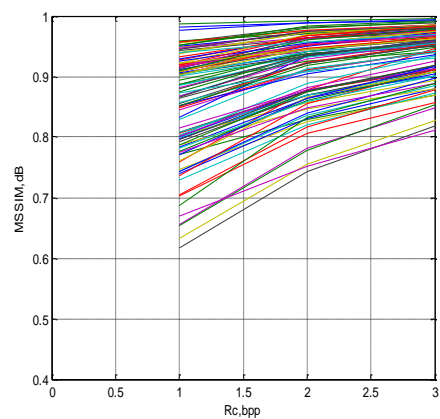

MSSIM

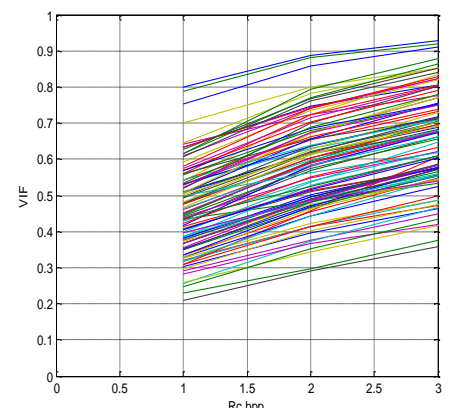

VIF

Figure 15. The variations of PSNR, MSSIM and VIF of 100 natural images

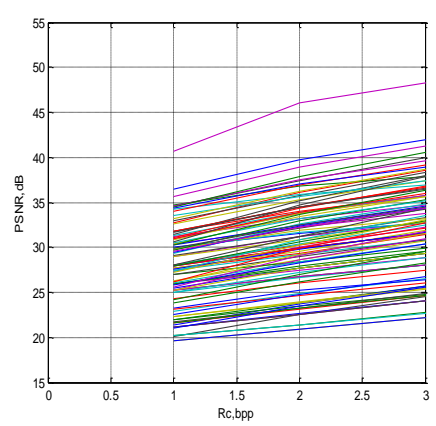

PSNR

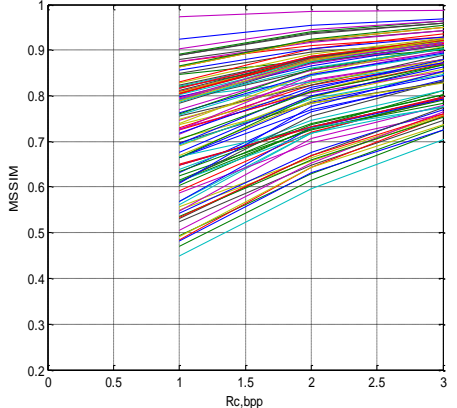

MSSIM

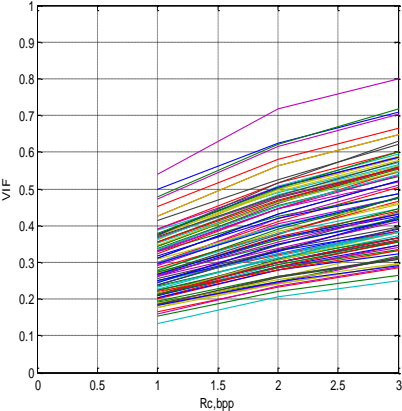

VIF

Figure 16. The variations of PSNR, MSSIM and VIF of 100 satellite images

Table 5 represents the resulting test Images by the selection algorithm, according to image types (medical, natural and satellite). In order to obtain the 20 test images, the applied thresholds on satellite images, enabled to choose 28 images from PSNR, 28 images from MSSIM and 47 images from VIF. For 20 natural test images, the selection algorithm selected 37 images from PSNR, 35 images from MSSIM and 51 images from VIF.

The selected images for medical images are, respectively, 36, 35 and 34. The test images are represented in Figures 17, 18, and 19. It can be observed that the loss in PSNR, MSSIM and VIF values is nearly constant whatever is the value of Rc for the three image types (medical, natural and satellite).

Figures 20, 21, and 22 represent the curves of PSNR, MSSIM and VIF for the 20 selected images (obtained by combaning both DWT+SPIHT-based and JPEG 2000-based selection algorithms) according to image types (medical, natural and satellite) using JPEG standard. It can be observed that the loss in PSNR, MSSIM and VIF values is nearly constant whatever is the value of Rc for the three image types (medical, natural and satellite). 
Table 5. PSNR, MSSIM and VIF thresholds

\begin{tabular}{|c|c|c|c|c|c|c|c|c|}
\hline \multicolumn{3}{|c|}{ Image Natural } & \multicolumn{3}{|c|}{ Image Medical } & \multicolumn{3}{|c|}{ Image Satellite } \\
\hline PSNR & MSSIM & VIF & PSNR & MSSIM & VIF & PSNR & MSSIM & VIF \\
\hline 5 & 1 & 1 & 5 & 1 & 1 & 5 & 1 & 1 \\
\hline 11 & 5 & 5 & 11 & 5 & 5 & 6 & 5 & 3 \\
\hline 14 & 8 & 11 & 14 & 8 & 11 & 10 & 6 & 5 \\
\hline 24 & 11 & 14 & 24 & 11 & 14 & 12 & 7 & 6 \\
\hline 25 & 14 & 15 & 25 & 14 & 15 & 15 & 8 & 7 \\
\hline 26 & 15 & 24 & 26 & 15 & 24 & 19 & 10 & 8 \\
\hline 29 & 23 & 25 & 29 & 23 & 25 & 21 & 12 & 9 \\
\hline 32 & 24 & 26 & 32 & 24 & 26 & 24 & 15 & 10 \\
\hline 34 & 25 & 32 & 34 & 25 & 32 & 25 & 18 & 12 \\
\hline 36 & 26 & 33 & 36 & 26 & 33 & 27 & 19 & 14 \\
\hline 37 & 32 & 34 & 37 & 32 & 34 & 28 & 21 & 15 \\
\hline 41 & 33 & 37 & 41 & 33 & 37 & 31 & 22 & 18 \\
\hline 42 & 35 & 41 & 42 & 35 & 41 & 32 & 24 & 19 \\
\hline 44 & 37 & 45 & 44 & 37 & 45 & 34 & 25 & 21 \\
\hline 45 & 40 & 49 & 45 & 40 & 49 & 36 & 27 & 22 \\
\hline 47 & 41 & 61 & 47 & 41 & 61 & 38 & 28 & 23 \\
\hline 48 & 44 & 63 & 48 & 44 & 63 & 40 & 34 & 24 \\
\hline 49 & 49 & 66 & 49 & 49 & 66 & 43 & 37 & 25 \\
\hline 57 & 60 & 67 & 57 & 60 & 67 & 46 & 40 & 27 \\
\hline 58 & 66 & 72 & 58 & 66 & 72 & 48 & 41 & 28 \\
\hline 61 & 67 & 73 & 61 & 67 & 73 & 74 & 42 & 30 \\
\hline 66 & 72 & 74 & 66 & 72 & 74 & 76 & 43 & 31 \\
\hline 67 & 73 & 76 & 67 & 73 & 76 & 78 & 45 & 32 \\
\hline 73 & 74 & 77 & 73 & 74 & 77 & 85 & 46 & 34 \\
\hline 76 & 76 & 78 & 76 & 76 & 78 & 86 & 47 & 35 \\
\hline 77 & 77 & 80 & 77 & 77 & 80 & 93 & 48 & 36 \\
\hline 78 & 78 & 81 & 78 & 78 & 81 & 95 & 60 & 37 \\
\hline 80 & 80 & 82 & 80 & 80 & 82 & 96 & 70 & 38 \\
\hline 81 & 81 & 83 & 81 & 81 & 83 & & 71 & 39 \\
\hline 82 & 82 & 84 & 82 & 82 & 84 & & 72 & 40 \\
\hline 83 & 83 & 88 & 83 & 83 & 88 & & 73 & 41 \\
\hline 84 & 84 & 91 & 84 & 84 & 91 & & 74 & 42 \\
\hline 85 & 91 & 96 & 85 & 91 & 96 & & 76 & 43 \\
\hline 88 & 96 & 100 & 88 & 96 & 100 & & 78 & 45 \\
\hline 91 & 100 & & 91 & 100 & & & 79 & 46 \\
\hline \multirow[t]{15}{*}{96} & & & 96 & & & & 81 & 47 \\
\hline & & & & & & & 83 & 48 \\
\hline & & & & & & & 84 & 71 \\
\hline & & & & & & & 85 & 74 \\
\hline & & & & & & & 86 & 76 \\
\hline & & & & & & & 88 & 77 \\
\hline & & & & & & & 89 & 78 \\
\hline & & & & & & & 93 & 79 \\
\hline & & & & & & & 95 & 84 \\
\hline & & & & & & & 96 & 85 \\
\hline & & & & & & & 97 & 86 \\
\hline & & & & & & & 99 & 93 \\
\hline & & & & & & & 95 & 95 \\
\hline & & & & & & & 96 & 96 \\
\hline & & & & & & & 99 & 99 \\
\hline
\end{tabular}

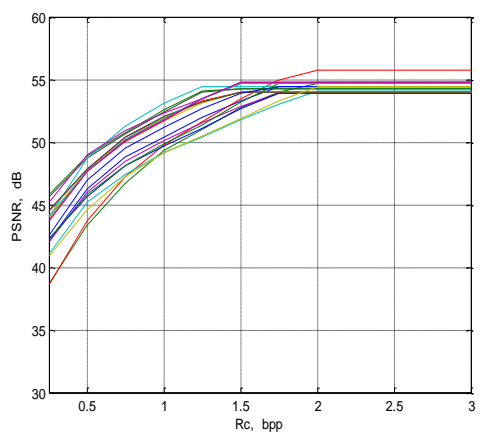

PNSR

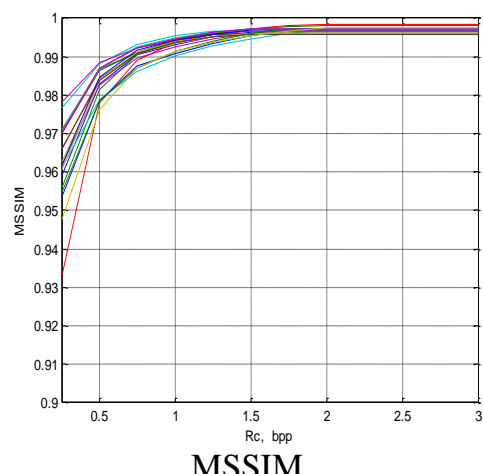

MSSIM

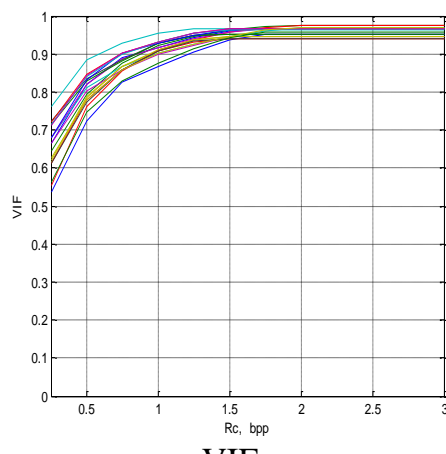

VIF

Figure 17. PSNR, MSSIM and VIF variations of 20 selected medical test images using JPEG 2000 standard 


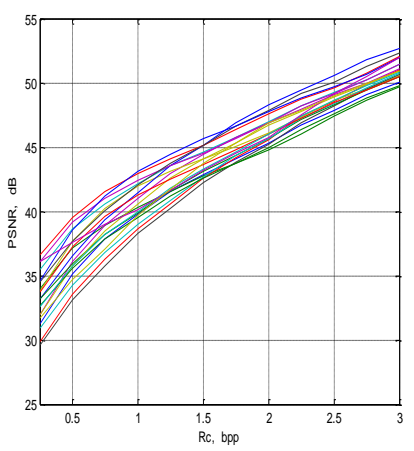

PNSR

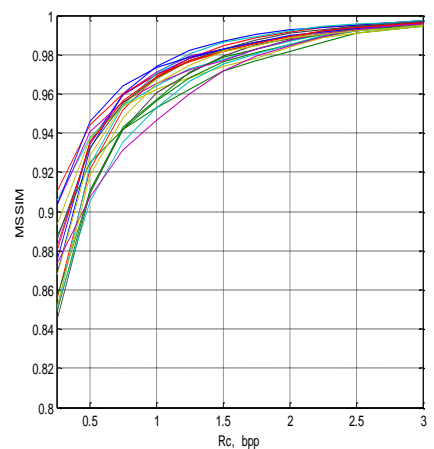

MSSIM

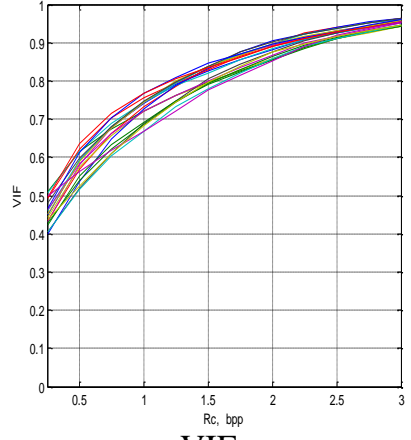

VIF

Figure 18. PSNR, MSSIM and VIF variations of 20 selected natural test images using JPEG 2000 standard

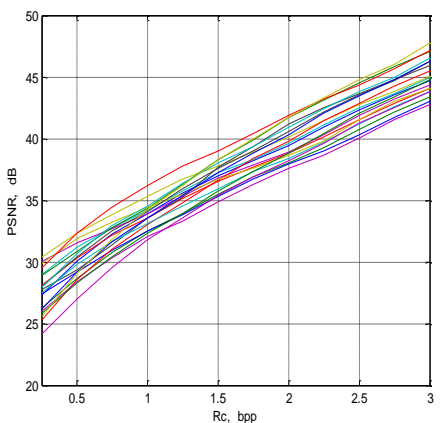

PNS

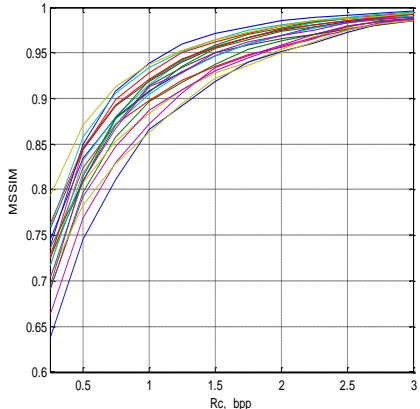

MSSIM

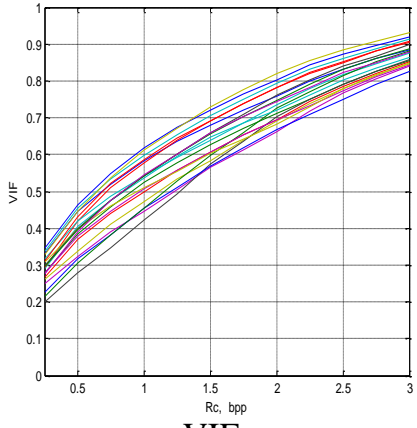

VIF

Figure 19. PSNR, MSSIM and VIF variations of 20 selected satellite test images using JPEG 2000 standard

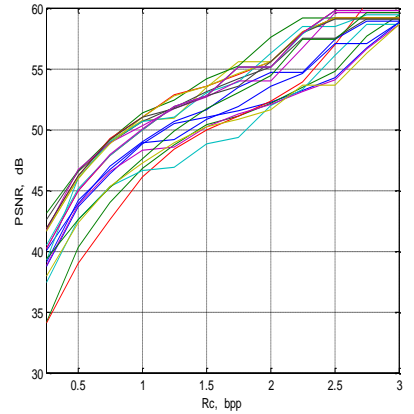

PNSR

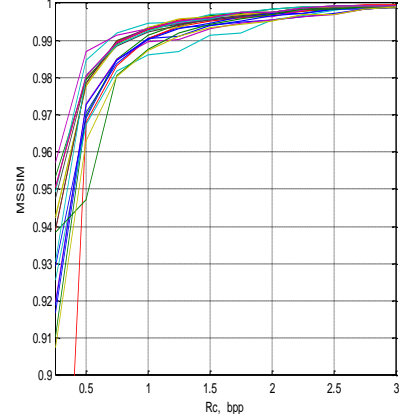

MSSIM

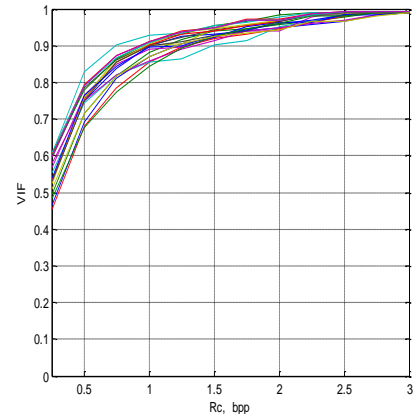

VIF

Figure 20. PSNR, MSSIM and VIF variations of 20 selected medical test images using JPEG standard

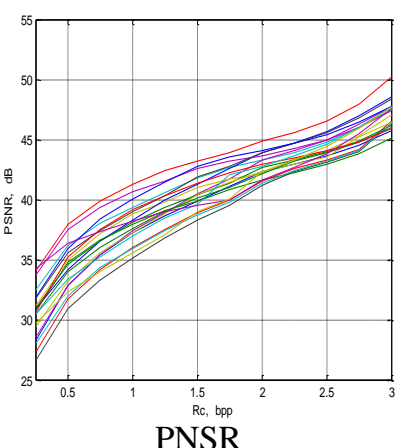

PNSR

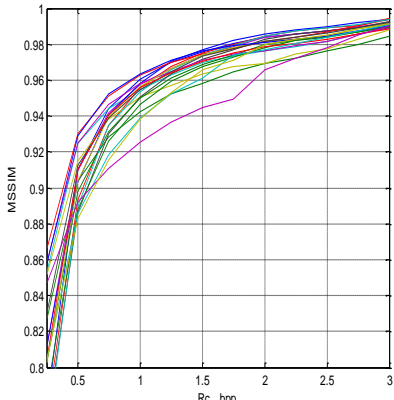

MSSIM

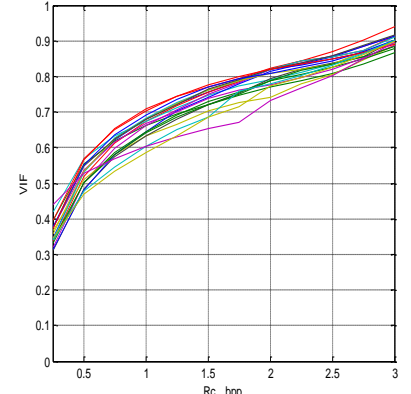

VIF

Figure 21. PSNR, MSSIM and VIF variations of 20 selected natural test images using JPEG standard 


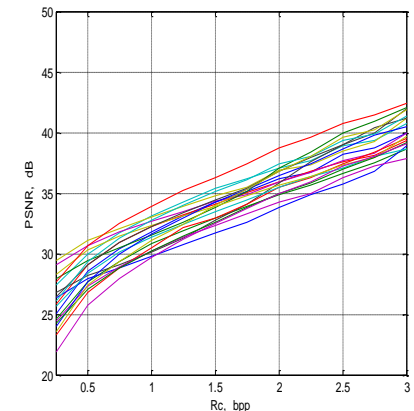

PNSR

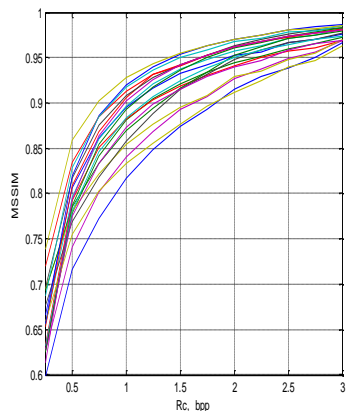

MSSIM

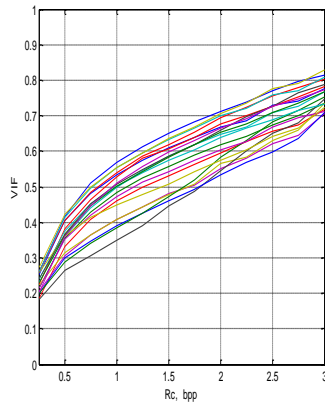

VIF

Figure 22. PSNR, MSSIM and VIF variations of 20 selected satellite test images using JPEG standard

Table 6 shows the PSNR, MSSIM, and VIF variance of the 20 images selected for each type (medical, natural, and satellite) after applying the algorithm used.

Table 6. The variances of PSNR, MSSIM, and VIF for the 20 selected images per iamge type

\begin{tabular}{cccccccccccc}
\hline & \multicolumn{3}{c}{ Satellite image } & \multicolumn{4}{c}{ Natural image } & \multicolumn{4}{c}{ Medical image } \\
Image & $\sigma_{\mathrm{PSNR}_{\mathrm{i}}}^{2}$ & $\sigma_{\mathrm{MSSIM}}^{2}$ & $\sigma_{\mathrm{VIF}_{\mathrm{i}}}^{2}$ & $\mathrm{Image}^{2}$ & $\sigma_{\mathrm{PSNR}_{\mathrm{i}}}^{2}$ & $\sigma_{\mathrm{MSSIM}_{\mathrm{i}}}^{2}$ & $\sigma_{\mathrm{VIF}_{\mathrm{i}}}^{2}$ & Image $^{2}$ & $\sigma_{\mathrm{PSNR}_{\mathrm{i}}}^{2}$ & $\sigma_{\mathrm{MSSIM}_{\mathrm{i}}}^{2}$ & $\sigma_{\mathrm{VIF}_{\mathrm{i}}}^{2}$ \\
\hline 5 & 0.22 & 0.0003 & 0.0023 & 9 & 2.11 & 0.0001 & 0.0001 & 5 & 0.81 & 0.0003 & 0.0020 \\
6 & 2.15 & 0.0002 & 0.0001 & 16 & 1.35 & 0.0004 & 0.0007 & 11 & 5.39 & 0.0001 & 0.0007 \\
10 & 0.64 & 0.0001 & 0.0012 & 21 & 3.46 & 0.0001 & 0.0004 & 14 & 2.93 & 0.0002 & 0.0022 \\
12 & 0.57 & 0.0005 & 0.0002 & 26 & 0.59 & 0.0002 & 0.0007 & 24 & 4.30 & 0.0001 & 0.0004 \\
15 & 1.32 & 0.0011 & 0.0040 & 30 & 1.11 & 0.0003 & 0.0002 & 25 & 1.23 & 0.0002 & 0.0011 \\
19 & 2.88 & 0.0002 & 0.0014 & 33 & 5.91 & 0.0001 & 0.0014 & 26 & 2.42 & 0.0002 & 0.0009 \\
24 & 2.80 & 0.0002 & 0.0045 & 39 & 2.20 & 0.0002 & 0.0014 & 32 & 4.80 & 0.0003 & 0.0014 \\
25 & 1.27 & 0.0018 & 0.0032 & 46 & 0.78 & 0.0000 & 0.0008 & 37 & 2.68 & 0.0001 & 0.0032 \\
34 & 4.89 & 0.0003 & 0.0027 & 59 & 1.46 & 0.0001 & 0.0008 & 41 & 5.35 & 0.0004 & 0.0012 \\
40 & 1.40 & 0.0007 & 0.0024 & 60 & 3.72 & 0.0000 & 0.0002 & 49 & 3.03 & 0.0003 & 0.0013 \\
43 & 0.83 & 0.0000 & 0.0005 & 61 & 0.18 & 0.0002 & 0.0008 & 57 & 5.01 & 0.0004 & 0.0047 \\
46 & 3.81 & 0.0002 & 0.0005 & 63 & 5.82 & 0.0001 & 0.0034 & 61 & 4.54 & 0.0004 & 0.0005 \\
48 & 1.58 & 0.0006 & 0.0008 & 67 & 3.12 & 0.0004 & 0.0011 & 66 & 2.55 & 0.0002 & 0.0011 \\
74 & 0.53 & 0.0007 & 0.0007 & 69 & 4.08 & 0.0002 & 0.0011 & 67 & 3.05 & 0.0003 & 0.0013 \\
76 & 0.80 & 0.0009 & 0.0053 & 73 & 1.75 & 0.0001 & 0.0010 & 73 & 1.39 & 0.0003 & 0.0028 \\
78 & 2.14 & 0.0005 & 0.0004 & 77 & 2.90 & 0.0003 & 0.0023 & 76 & 0.68 & 0.0002 & 0.0009 \\
85 & 5.06 & 0.0011 & 0.0030 & 84 & 0.86 & 0.0002 & 0.0013 & 78 & 2.46 & 0.0004 & 0.0032 \\
86 & 0.73 & 0.0012 & 0.0043 & 85 & 0.11 & 0.0002 & 0.0014 & 80 & 3.06 & 0.0004 & 0.0032 \\
93 & 5.72 & 0.0001 & 0.0002 & 88 & 2.41 & 0.0004 & 0.0014 & 82 & 1.84 & 0.0003 & 0.0023 \\
96 & 2.58 & 0.0018 & 0.0054 & 93 & 0.96 & 0.0001 & 0.0007 & 91 & 3.10 & 0.0001 & 0.0006 \\
\hline
\end{tabular}

To better evaluate the effectiveness of the selection algorithm, we traced on Figures 23, 24, and 25 the curves of the variance of each evaluation parameter for all types of images. It can be seen that a slight difference between the three compression algorithms; which makes it possible to say that the selected images are adequate for testing other image compression algorithms based on a transformation.

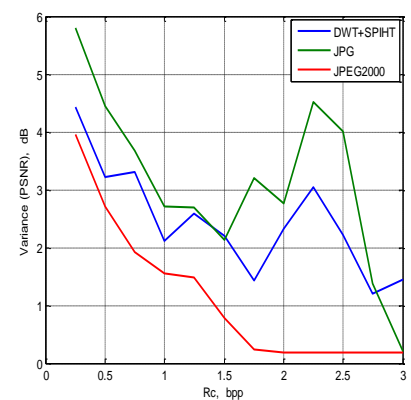

PNSR

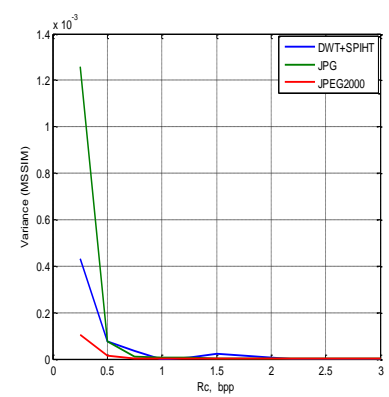

MSSIM

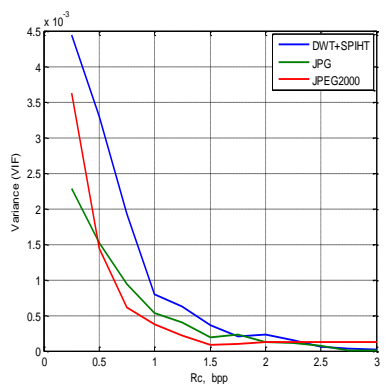

VIF

Figure 23. The variation of the variance of PSNR, MSSIM and VIF for the 20 selected medical test images 


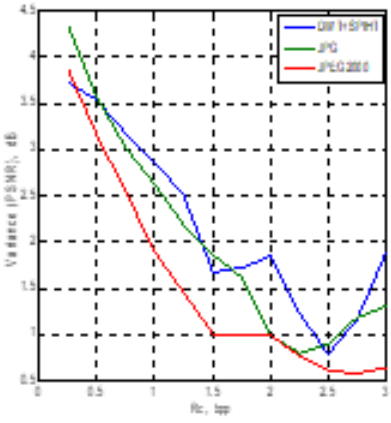

PNSR

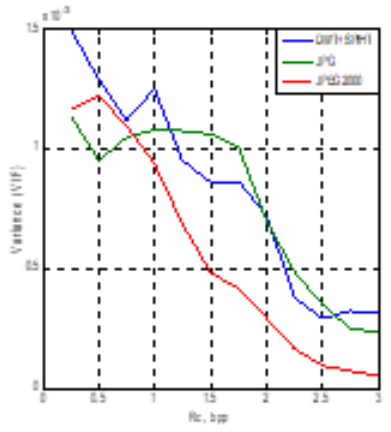

MSSIM

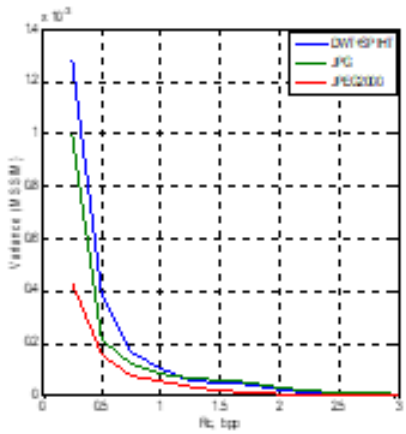

VIF

Figure 24. The variation of the variance of PSNR, MSSIM and VIF for the 20 selected natural test images

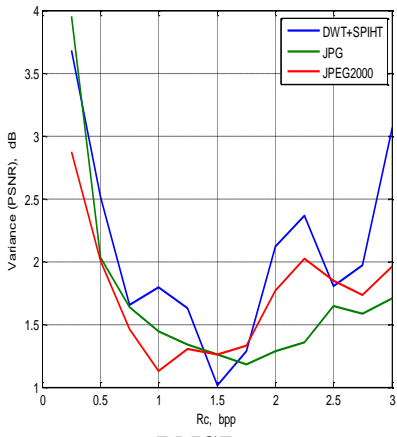

PNSR

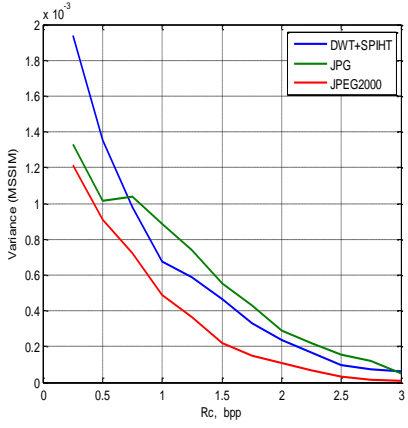

MSSIM

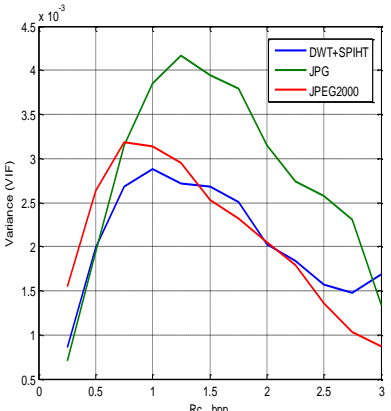

VIF

Figure 25. The variation of the variance of PSNR, MSSIM and VIF for the 20 selected satellite test images

\section{CONCLUSION}

In this research work, we proposed a selection algorithm of $\mathrm{M}$ images based on statistics calculated from images evaluation parameters (PSNR, MSSIM and VIF) according to choices of variances thresholds $\mathrm{S}_{\mathrm{PSNR}}, S_{M S S I M}$ and $S_{V I F}$. We also applied the same algorithm of selection using three compression algorithms namely DWT+SPIHT, JPEG and JPEG2000. As perspective, we are going to apply the same algorithm of selection and compare the results including other methods of compression (wavelet-packet transform [17, 18] and quincunx transform [19-21]).

\section{REFERENCES}

[1] E. Sjöblom, Compression of Medical Image Stacks using Wavelets and Zero Tree Coding", Master thesis, Division of Image Coding, Department of Electrical Engineering, Linköping University junry, 2002.

[2] M.Beladgham et al., "Improving Quality of Medical Image Compression Using Biorthogonal CDF Wavelet Based on Lifting Scheme and SPIHT Coding", serbian journal of electricatl engineering, vol. 8(2), pp. 163-179, may 2011.

[3] M. Beladgham, Construction d'une technique d'aide au diagnostic en imagerie médicale. Application a la compression d'images, Doctoral thesis, University of Tlemcen, Dec 2012.

[4] T. Kavitha and K. Jayasankar, "Ideal Huffman Code for Lossless Image Compression for Ubiquitous Access," IJEECS (Indonesian Journal of Electrical Engineering and Computer Science), vol. 12(2), Nov 2018.

[5] D. Taubman and M. Marcellin, JPEG2000: Image compression fundamentals, standards and practice Boston, Nov. 2001.

[6] Ph. Corvisier, "La Norme JPEG Adopte les Ondelettes," Revue Electronique, (9), pp. 15-165, Sep 1999.

[7] A. Trémeau, C. Fernandez-Maloigne \& P. Bonton, Image Numérique Couleur, de l'Acquisition au Traitemen", Ed. DUNOD, 1984

[8] C. Herley and M. Vetterli, "Wavelets and recursive filter banks," IEEE Transaction on Signal Processing, vol. 41(8), pp. 2536-2556, Aug 1993.

[9] CL. Guinet and J. Grellet, Introduction à l'IRM De La Théorie A La Pratique, Ed. Masson, 1992.

[10] M. Beladgham et al., "Medical Image Compression Using Quincunx Wavelets and SPIHT Coding," Journal of Electrical Engineering \& Technology, vol. 7(2), pp. 264-272, 2012. 
[11] H. R. Sheikh and A. C. Bovik, "Image Information and Visual Quality," IEEE Transaction on Image Processing, pp. 430-444, Sep 2004.

[12] E. Dumic, S. Grgic \& M. Grgic, "New Image-Quality Measure Based on Wavelets," Journal of Electronic Imaging, vol. 19(1), pp. 011-018, Mar 2010.

[13] A. K. Moorthy, Z. Wang \& A. C. Bovik, Visual Perception and Quality Assessment, Chapiter19 in Optical and digital image processing, Ed. Wiley, 2010.

[14] Z. Wang and Q. LI, "Information Content Weighting for Perceptual Image Quality Assessment," IEEE Transactions on Image Processing, vol. 20(5), pp. 1185-1198, May 2011.

[15] S. Dubuisson, D. Béréziat \& N. Thome, Bases du traitement des images - Opérations de Base et Améliorations, 2016.

[16] N. T. Tankam., Traitement Numérique d'Images et Applications : Méthodes statistiques optimisées pour le traitement numérique des images de grandes tailles, Ed. Editions Universitaires Européènne, France, 2015.

[17] I. Benyahia, M. Beladgham \& A. Bassou," Evaluation of the Medical Image Compression using Wavelet Packet Transform and SPIHT Coding", IJECE (International Journal of Electrical and Computer Engineering), vol. 8(4), 2018.

[18] M. Latfaoui and F. Bereksi Reguig, "Packets Wavelets and Stockwell Transform Analysis of Femoral Doppler Ultrasound Signals," IJECE (International Journal of Electrical and Computer Engineering), vol. 8(6), Dec 2018.

[19] F. Manuela, VD. Dimitri, U. Michael, "An Orthogonal Family of Quincunx Wavelets With Continuously Adjustable Order," IEEE Transactions on Image Processing, vol. 14(4), Apr 2005.

[20] V. D. Dimitri, B. Thierry \& U. Michael, "On the Multidimensional Extension of the Quincunx Subsampling Matrix", IEEE Signal Processing Letters, vol. 12(2), Feb 2005.

[21] Y. Chen, A. D. Michael \& L. Wu-Sheng, "Design of Optimal Quincunx Filter Banks for Image Coding," EURASIP Journal on Advances in Signal Processing, 2007. 